\title{
Torsional Dependence of the Critical Current in 2G Tapes
}

\author{
G.G. Sotelo, X. Granados, M. Carrera, J. Lopez Lopez
}

Abstract- Since the second generation (2G) superconducting wires started to be produced in a large scale almost a decade ago, the quality of the materials manufactured has been continually improved. Every year the manufacturers present new materials with better electrical properties, increasing the critical current density, critical magnetic field and the total length produced with continuous homogeneity. The mechanical properties of the coated conductor should be taken in account, since electro-dynamical forces appear in working conditions as stress in the cooling and heating processes. In applications as fault current limiters, SMES, transformers, electromagnets, motors, cables, etc., the consideration of them is mandatory to the project of those devices. In this context, the present work studies the electrical and mechanical properties of $12 \mathrm{~mm} \mathrm{2G}$ wires samples under torsion without axial tension. Critical current is measured as a function on the torsion angle. Details of the experiment and their results are here presented.

Index Terms- 2G superconducting wire, coated conductor, Twisted wire, superconducting power cable.

\section{INTRODUCTION}

The second generation (2G) wires represent a revolution for the superconductivity large scale applications. Nowadays there are at least 5 factories that promise to produce high homogeneity $2 \mathrm{G}$ wires with hundreds of meters [1-3]. Considering the coated conductors applications for electrical power system, the fault current limiters and the cables for energy transmission are technology with higher level of maturity, and they are also becoming commercial products [4-9]. Notwithstanding, the investigation of those devices is the goal of several international research groups in order to improve the design and performance of these equipments.

Aiming to power cable manufacturing with $2 \mathrm{G}$ wire, several topologies are being considered in order to approach transposition: CORC (Conductor on Round Core) [10,11], Roebel [12,13], Helicoidal [14] and twisted stacked wires [15-17]. Between all proposals suggested, twisted stacked wires, appear as the simplest design. In our opinion this configuration is one of the more versatile topologies available and it could have the best benefit/cost relation.

Considering then the development and characterization of the twisted staked wires, the present paper points the study of the behavior of a single wire when twisted. The critical current of a $12 \mathrm{~mm}$ wide wire sample was measured for several torsion angles avoiding any axial tension. The study of the critical current dependency with the angular pitch was presented previously in the literature for the $4 \mathrm{~mm}$ wires [18-20], but it was not done yet for $2 \mathrm{G}$ wires with $12 \mathrm{~mm}$ width. In the present paper a $12 \mathrm{~mm} \mathrm{2G}$ sample wire was tested. Besides the $\mathrm{V}(\mathrm{I})$ measurements, the external magnetic induction produced the by the $2 \mathrm{G}$ sample wire was scanned with a Hall probe for several angular positions. The goals of these measurements are: (a) the influence of pure twisting on the current density distribution according to the pitch angle, and (b) the HTS limits introduced by non linear deformation as buckling appear in a $2 \mathrm{G}$ twisted wire. 


\section{EXPERIMENTAL RIg DESCRIPTION}

In this section it will be presented the details about the experimental apparatus and the procedures to measure a $2 \mathrm{G}$ wire superconducting sample. The sample was produced by SuperOx [21], and its details are shown in Table I.

TABLE I. 2G WIRE SAMPLE SPECIFICATIONS

\begin{tabular}{ll}
\hline \hline Parameter & Value \\
\hline $\begin{array}{l}\text { Superconducting } \\
\text { Material }\end{array}$ & RE-Ba-Cu-O \\
Year of production & 2015 \\
Commercial name & $\begin{array}{l}\text { SuperOx 12-20Ag-20Cu- } \\
\text { WOH }\end{array}$ \\
Width & $12 \mathrm{~mm}$ \\
Total thickness & $110 \mu \mathrm{m}$ \\
Substrate & Hastelloy C-276 (60 $\mu \mathrm{m})$ \\
Cu Layer thickness & $20 \mu \mathrm{m}$ (each one) \\
Ag-Layer thickness & $2 \mu \mathrm{m}$ \\
HTS thickness & $1-2 \mu \mathrm{m}$ \\
\hline \hline
\end{tabular}

The sample holder presented in Fig. 1 was designed in order to measure the dependence of the critical current on the angular pitch of the torsion. The electrical schematic and wire dimensions are presented in Fig. 2. To rotate the wire, swivel connectors were turned synchronized with the same angular displacement amplitude $\alpha$, but in opposite directions, generating so a twisting angle $2 \alpha$. In order to measure the magnetic induction $\mathrm{Bz}(\mathrm{x}, \mathrm{y})$ produced by the transport current, a Hall probe set of $30 \mathrm{~mm}$ long $x$ axis scans was done covering an area of $3 \mathrm{~mm}$ around the center of the tape along the $y$ axis. The Hall sensor used here has an active area of $100 \times 100 \mu \mathrm{m} 2$.

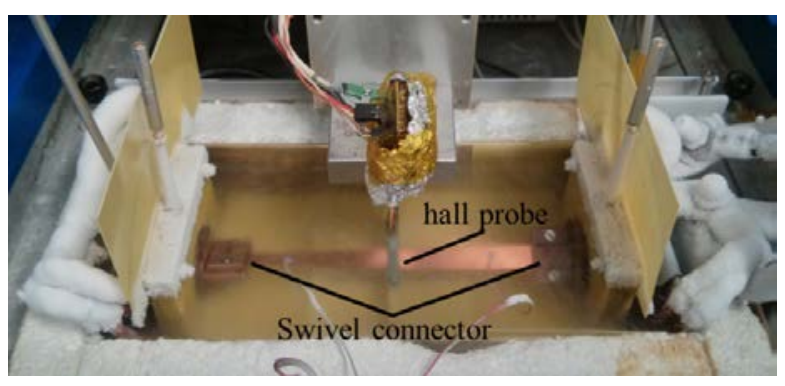

Fig. 1. Experimental rig designed to measure the electrical properties of the $2 \mathrm{G}$ wire and the external field produced by it. 


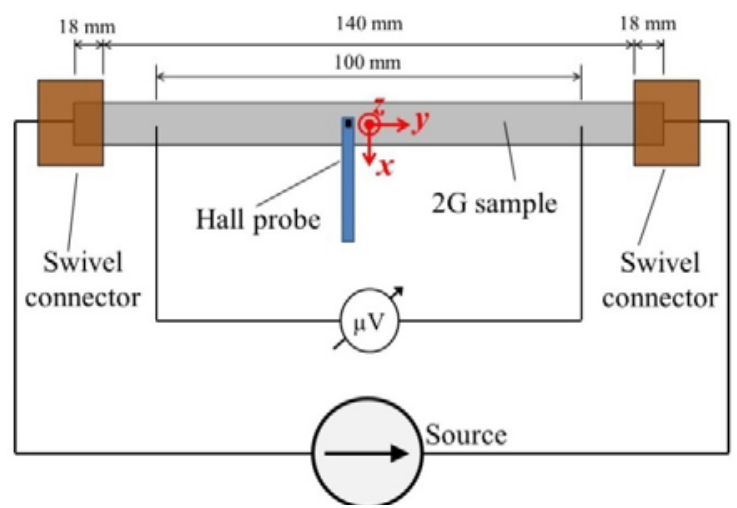

Fig. 2. Electric connections used in this experiment.

The experiments were made in the following sequence: the sampled was cooled in LN2 waiting for approximately 15 minutes for reaching the thermal equilibrium. The angular position was fixed for the wire; a constant transport current, I, was applied and some minutes were waited avoiding so the transitory and the flux creep effects; finally, the map was made above the surface. Then the current was increased to a new constant value, and the magnetic induction was mapped again. This procedure was repeated several times until reach a current equal to $I_{c}$, determined by the $1 \mu \mathrm{V} / \mathrm{cm}$ criterion. After reaching $I_{c}$, the current was reduced to $I=0 \mathrm{~A}$, and a field map was made in the remnant state of the sample. Finally, the angular position was changed and all the procedure described above was repeated.

\section{BUCKLING}

When a flat wire is twisted, it is subjected to buckling effects, depending on the twisting angle due to the compressive stress in central area of the tape generated by the twisting. The buckling is well known in mechanical engineering $[22,23]$, but it wasn't discussed in the $2 \mathrm{G}$ wire literature. Some authors impose an axial load in order to avoid buckling $[18,19]$ thinking in arising a higher pitch before it appears, during the tests with the $4 \mathrm{~mm}$ width wire. In the present work no axial load was applied to the wire, and the buckling was observed in the sample after a pitch of $0.6 \% / \mathrm{mm}$, approximately. In Fig. 3 , it is shown the Buckling observed in the sample for $90,120,150$ and180 twisted degrees. Those are some of the twisting angles applied to the $2 \mathrm{G}$ wire during the tests presented in the sequence of the experiments. 


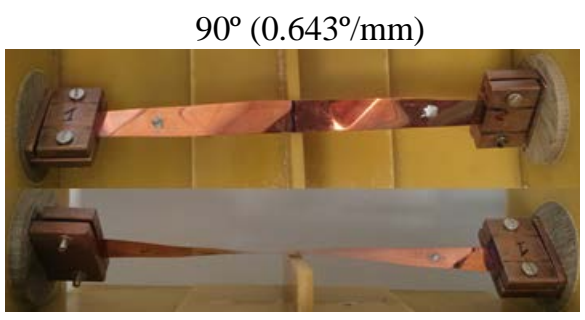

$120^{\circ}\left(0.857^{\circ} / \mathrm{mm}\right)$

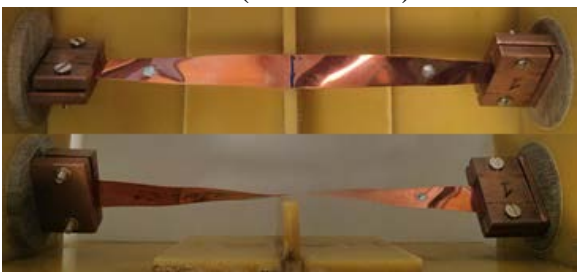

$150^{\circ}\left(1.071^{\circ} / \mathrm{mm}\right)$

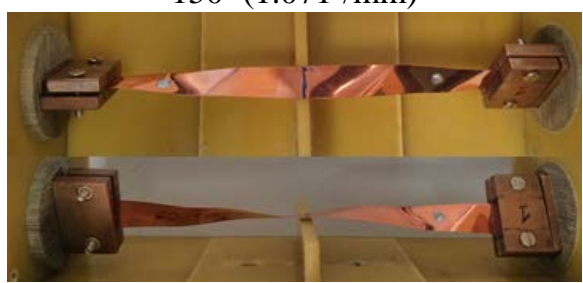

$180^{\circ}\left(\right.$ or $\left.1.286^{\circ} / \mathrm{mm}\right)$

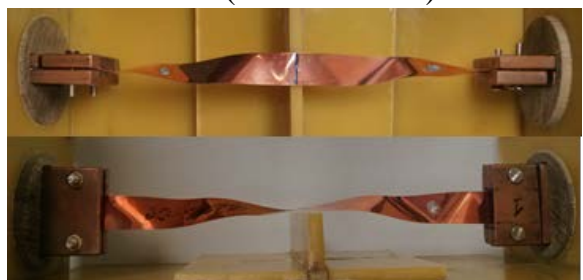

Fig. 3. Twisting of a $12 \mathrm{~mm} 2 \mathrm{G}$ sample. Buckling appears for angles larger than 120 in absence of axial tension, relaxing so the compressive stress in the central-longitudinal region. The central-transversal line marks de route of the Hall probe scanning .

\section{Twisting Angle Dependency of the Critical Current}

The results presented in the following two sections have been obtained by applying the experimental procedure described in section II. For each measured twisting angle, the V(I) curve of the sample was obtained by conventional current scan procedure well beyond the 1 $\square \mathrm{V} / \mathrm{cm}$ threshold, in order to obtain accurate values of the critical current $\left.I_{(}\right)$and the $n$ exponent. Fig 4 presents the results for the wire flat, before and after twisting experiments. $\mathrm{V}(\mathrm{I})$ characterization was made for each measured twisted angle, and $I_{c}$ and $n$ exponent were obtained by the fitted data for the power-law.

During the experiments, for a maximal rotation of 180 을 in a sample with $140 \mathrm{~mm}$ (or 1.286 o/mm), no significant change of the critical current and the $n$ exponent was observed, as presented in Figs. 5 (a) and (b), respectively. The critical current measured in wire for the case without torsion was $414 \mathrm{~A}$.

We expected that, beyond a certain pitch, the torsion applied to the sample would damage it since a permanent deformation in the buckled areas was observed after the experiment. No change in the critical current, however, could be detected. Probably it did not happen because no axial tension was applied to the sample during the angular torsion. Axial tensions increase the tensile strain in the lateral areas of the tape achieving the permanent damage strain threshold at lower pitch values. 


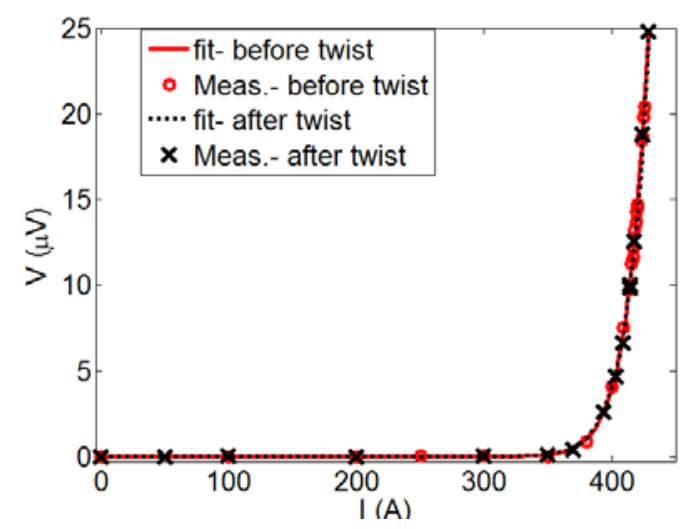

Fig. 4. V(I) curves for the flat, before and after twisting.

a)

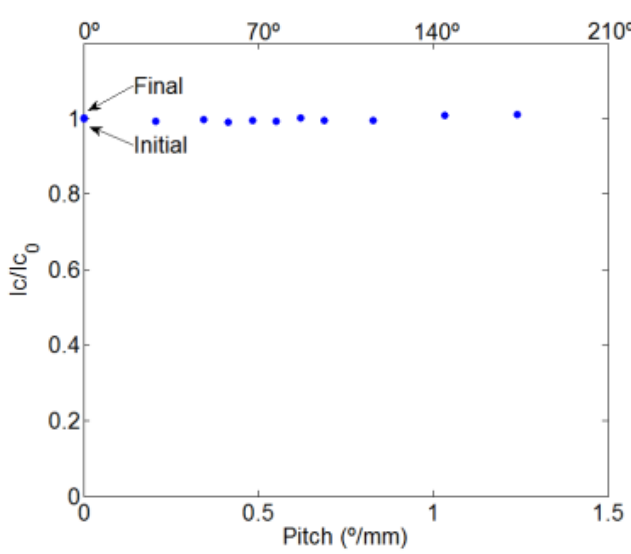

b)

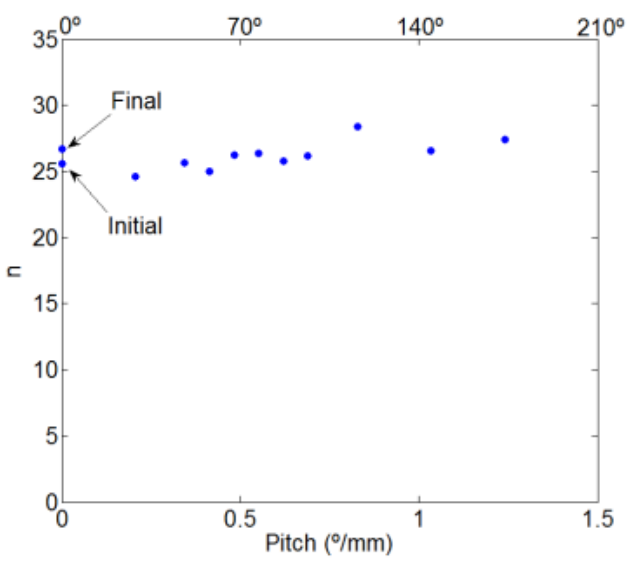

Fig. 5. Normalized critical current (a) and $n$ exponent as a pitch function.

\section{HALl PROBE SCANNING EXPERIMENTS WITH TRANSPORT CURRENT IN THE TWISTED WiRE}

The magnetic flux density $B_{z}(x, y)$ was measured above the sample surface in order to analyze the possible current distribution change in the wire due to the strain changes along the $x$ axis. We show in the following results (Figs. 6 to 11) the measured magnetic induction $B_{z}(x)$ across the central $y$ position (Fig. 2). The position $x=0 \mathrm{~mm}$ represents the central position of the wire.

Fig. 6 presents $x$-axis scans of the magnetic induction $z$ component profiles produced by the wire without torsion for several applied currents, from $50 \mathrm{~A}$ to $I_{c}=414 \mathrm{~A}$. It can be observed that the central region of the wire presents almost zero magnetic field for the lower currents. It indicates that the current flows only along the wire borders as expected, in accordance with the penetration process in a superconductor. For currents larger than $200 \mathrm{~A}, B_{z}$ profile starts to be asymmetric, suggesting that more current is flowing through the left half $(-6-0 \mathrm{~mm})$, than through the right half $(0-6 \mathrm{~mm})$. It habitually corresponds to inhomogeneity of the tape, When the current is closer to $I_{c}$, clear distortions of the profile appear (as in the region between $x=1-3 \mathrm{~mm}$ in Fig. 6 , for the $400 \mathrm{~A}$ and $414 \mathrm{~A}$ ) as a consequence of the possible inhomogeneity.

The same test was made for a $0.643 \% / \mathrm{mm}$ pitch (90을 rotion), as presented in Fig. 7 . The distortions observed in the right side are more evident.

Although the trends are similar, as fundamental difference, the peaks become narrower and the asymmetry in the magnetic induction profile is higher. 


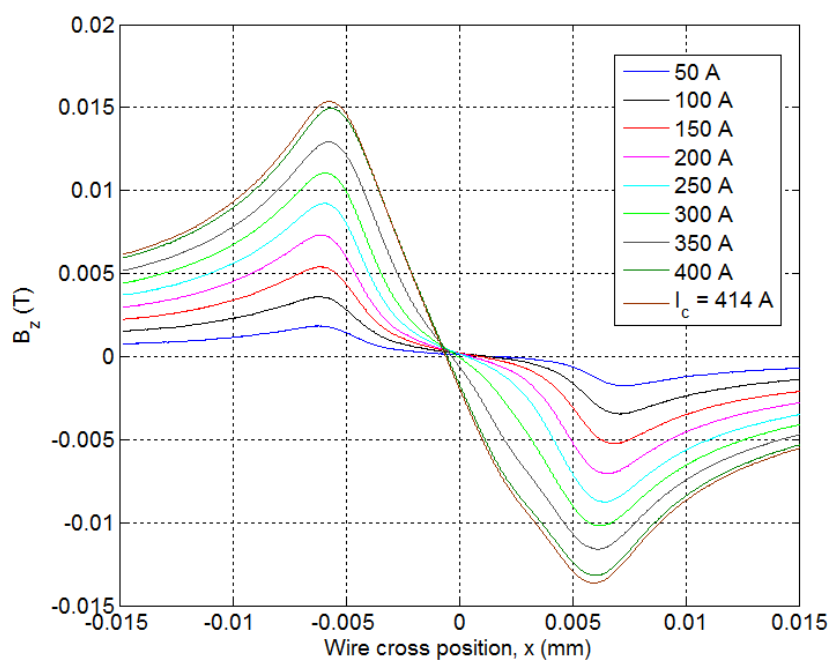

Fig. 6. Measured z-axis magnetic induction along $x$-axis scans on wire without torsion.

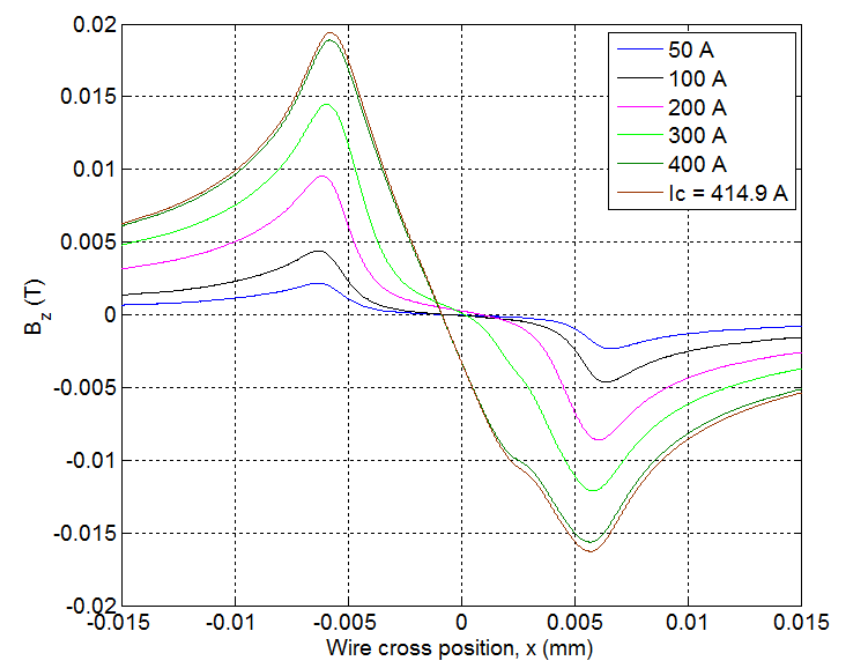

Fig. 7. Measured z-axis magnetic induction along $x$-axis scans for a rotation of $900(0.643$ o/mm).

That behavior can be observed in the set of scan profiles in Fig. 8 where the $B_{z}(x)$ profiles for $I=100 \mathrm{~A}$ are presented as a function of the torsional angle. Without torsion, the magnetic profile is practically symmetric. After twist the wire, this symmetry is lost.

Almost the same result can be observed for a transport current of $\mathrm{I}=200 \mathrm{~A}$, as presented in Fig. 9. The main difference now is that the magnetic field is more asymmetric, since the magnitude of the peak in the left size is higher than the absolute value on the right size.

Fig. 10 present the result for a transport current of $I=400 \mathrm{~A}$. This value is equal to $0.97 I_{c}$ of the sample. A $B_{z}(x)$ profile distortion around $x=2.5 \mathrm{~mm}$ is noted when the sample is twisted for pitch higher than $0.214 \% / \mathrm{mm}$. This distortion in the field can't be attributed to the buckling effect, since it starts to be observed for pitches in the range of $0.6 \% / \mathrm{mm}$. 


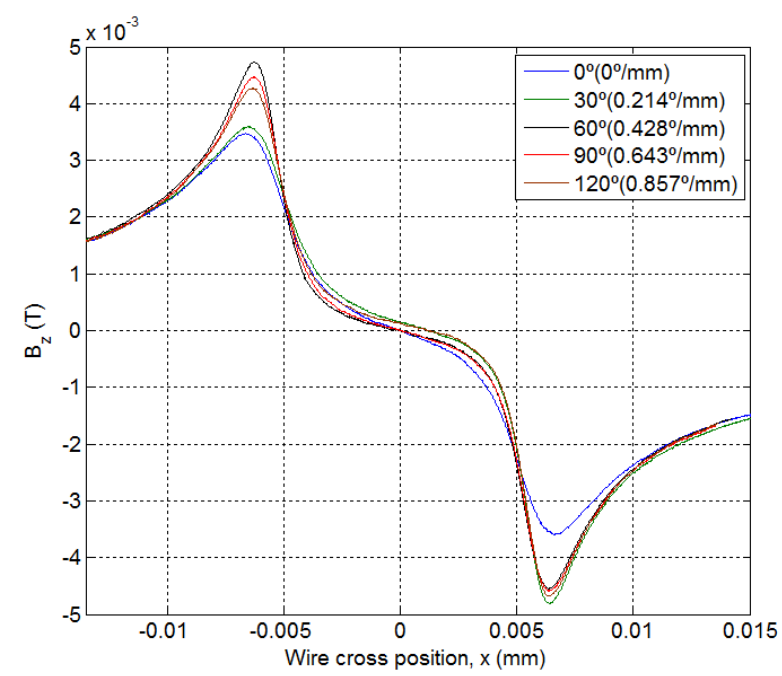

Fig. 8. Magnetic induction profile for several torsional angles and a transport current of 100A.

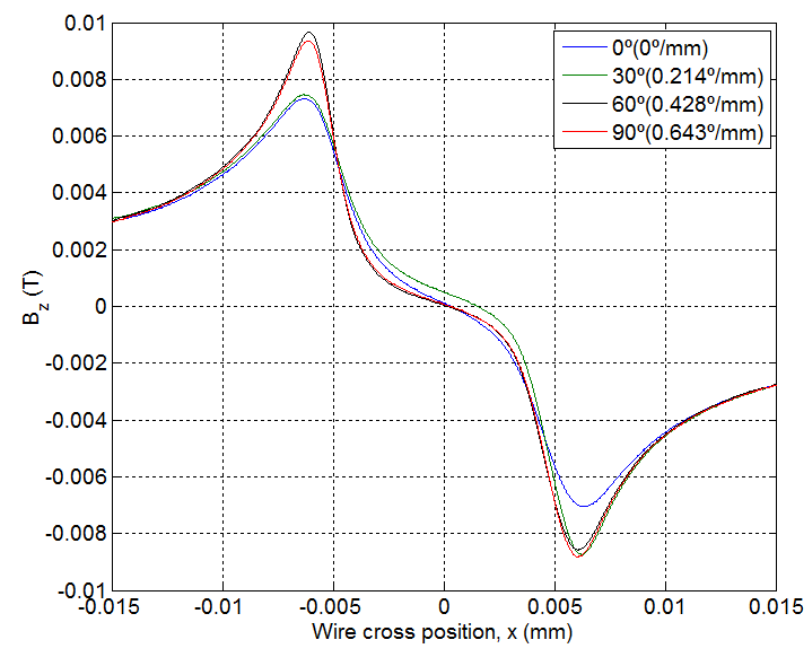

Fig. 9. Magnetic induction profile for several torsional angles and a transport current of $200 \mathrm{~A}$.

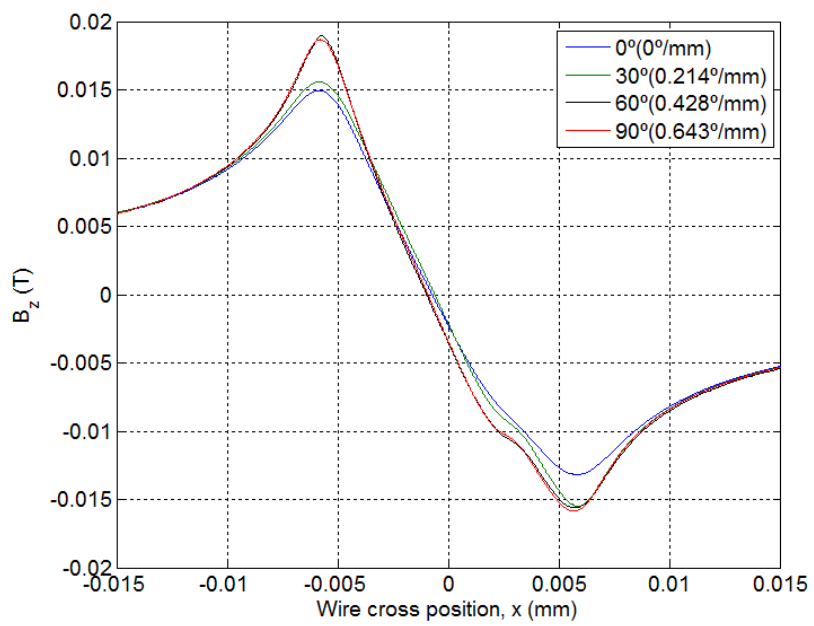

Fig. 10. Magnetic induction profile for several torsional angles and a transport current of 400A. 
Finally, Fig. 11 presents the magnetic field profile in the wire after the transport current was reduced to zero. By the $B_{z}(x)$ profile, it should be verified that two persistent currents loops with opposite directions that are circulating into the sample also have asymmetries. The distortion observed for the $B_{z}(x)$ profile is not observed for those results.

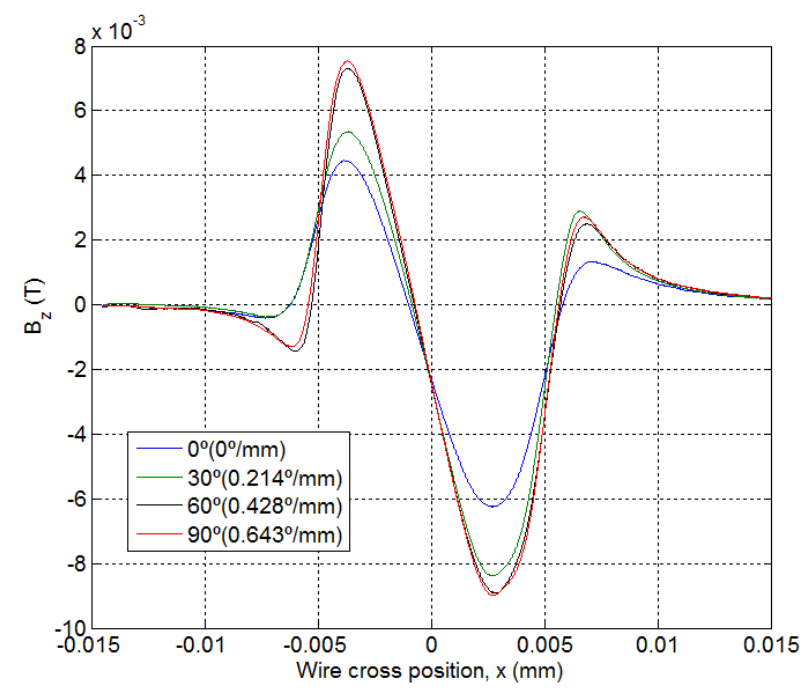

Fig. 11. Magnetic induction profile for the remnant field and several torsional angles.

\section{CONCLUSION}

This work studied pure torsional effects in a $12 \mathrm{~mm} \mathrm{HTS} 2 \mathrm{G}$ commercial wire. A Hall probe mapping system has been adapted for application to direct measurements over twisted wires. The system is able to explore the transport current distribution on the wire.

In the experiments, no axial load was imposed to the wire, thus allowing buckling in the sample for pitches higher than $0.6 \% / \mathrm{mm}$.

We observed that the $2 \mathrm{G}$ wire did not degrade with the twisting even when it was submitted to torsional angles where the buckling effects were present.

In-homogeneities observed previously to the twisting process are also detected in the twisted situation at the same level of current, showing so that these defects do enhance by the mechanic stress induced during twisting.

No significant variation of $I_{c}$ and $n$ exponent, obtained from $V(I)$ measurements, was observed for a maximum pitch of $1.286 \% / \mathrm{mm}$.

The next steps of this work will be: to repeat the experiment with $2 \mathrm{G}$ wires made from different manufactures in order to verify if they have the same behavior and to measure the magnetic field in $2 \mathrm{G}$ wires under simultaneous torsion and axial tension. Those measurements can help for the future modeling of the twisted stacked wire.

\section{ACKNOWLEDGMENTS}

This work was supported in part by EU (EU FP7-2013-ICT-609029 FORTISSIMO project, EU-FP7 NMP-LA-2012-280432 EUROTAPES project, Eurofusion PPPT-WPMAG 2014 and EU COST COST ACTIONS MP1201and MP1014), MINECO (RTC-2014-1740-3 and MAT2014-51778-C2-1-R), Generalitat de Catalunya (Xarmae) and to CNPq (Ciencia sem Fronteiras).

G.G. Sotelo is with the Fluminense Federal University (UFF) and the Materials Science Institute of Barcelona (CSIC-ICMAB). Adress: Rua Passo da Patria, 156, Niterói - RJ, CEP.: 24210-240, Brazil (Phone: +55 21 2629-5358, e-mails: gsotelo@id.uff.br / gsotelo@icmab.es). 
X. Granados is with the Materials Science Institute of Barcelona (CSIC-ICMAB). Adress: Campus UAB, Cerdanyola del Vallès, 08193, Barcelona, Spain. (Phone: +34935801853 , e-mail: granados@icmab.es).

M. Carrera is with Dep. Medi Ambient i Ciències del Sòl, Universitat de Lleida (UdL). Adress: Jaume II, 69. 25001 Lleida, Spain (e-mail: mcarrera@macs.udl.cat).

J. Lopez Lopez is with Dep. Física i Enginyeria Nuclear, Universitat Politècnica de Catalunya (UPC). Address: Edifici Euetib (CEIB), Despatx S11a, Comte D'urgell, 187, 08036 Barcelona, Spain (e-mail: josep.lopez-lopez@upc.edu)

\section{REFERENCES}

[1] X. Obradors and T. Puig: "Coated Conductors For Power Applications: Materials Challenges," Supercond. Sci. Technol., 27 (4), art. no. 044003, 2014.

[2] A. M. WOLSKY: "HTS from Pre-Commercial to Commercial: A Roadmap to Future Use of HTS by the Power Sector," Paris: International Energy Agency, 28 p., 2013.

[3] S. Nishijima, et. al.: "Superconductivity and the environment: a Roadmap," Supercond. Sci. Technol. 26, art. no. 113001, 2013.

[4] J. Bock, et. al.: "Resistive Superconducting Fault Current Limiters Are Becoming a Mature Technology," IEEE Trans. on Appl. Supercond., v. 25 (3), pp.1-4, June, 2015.

[5] L. Martini, et. al.: "Live Grid Field-Testing Final Results of the First Italian Superconducting Fault Current Limiter and Severe 3-Phase Fault Experience," IEEE Trans. on Appl. Supercond., v. 25 (3), pp.1-4, June, 2015.

[6] S. Elschner, el. al.: "ENSYSTROB: design, manufacturing and test of a 3-phase resistive fault current limiter based on coated conductors for medium voltage application," Physica C: Superconductivity, v. 482, pp. 98-104, nov. 2012.

[7] J. F. Maguire, et. al.: "Development and demonstration of a HTS power cable to operate in the long island power authority transmission grid," IEEE Trans. on Appl. Supercond., v. 17 (2), pp.2034-2037, June, 2007.

[8] S. Honjo, et. al.: "Electric properties of a $66 \mathrm{kV} 3$-core superconducting power cable system," IEEE Trans. on Appl. Supercond., v. 13 (2), pp. 1952-1955, June, 2003.

[9] M. Yagi, et al.: "Experimental Results of 275-kV 3-kA REBCO HTS Power Cable," IEEE Trans. on Appl. Supercond., v. 25 (3), pp. 1-5, art. no. 5401405, June, 2015.

[10] M. Majoros, et al.: "Magnetization losses in superconducting YBCO conductor-onround-core (CORC) cables," Supercond. Sci. Technol., 27 (12), art. no. 125008, 2014.

[11] F. Gömöry, et. at.: "Round Conductor With Low AC Loss Made From High-Temperature Superconducting Tapes," IEEE Trans. on Appl. Supercond., v. 25 (3), pp.1-4, June, art. no. 8201004, 2015.

[12] W Goldacker, et al.: "Roebel cables from REBCO coated conductors: A one-century-old concept for the superconductivity of the future" Supercond. Sci. Technol., vol. 27 (9), art. no. $093001,2014$.

[13] J. Fleiter, et al.: "Characterization of roebel cables for potential use in high-field magnets," IEEE Trans. on Appl. Supercond., v. 25 (3), pp.1-4, June, art. no. 6945872, 2015. 
[14] N. Amemiya, et al.: "Ac loss reduction of multilayer superconducting power transmission cables by using narrow coated conductors," Supercond. Sci. Technol., vol. 24 (6), art. no. 065013, 2011.

[15] Makoto Takayasu, et al.: "Conductor Characterization of YBCO Twisted Stacked-Tape Cables," IEEE Trans. on Appl. Supercond., v. 23 (3), pp.1-4, June, art. no. 4800104, 2013.

[16] L. Chiesa, N. C. Allen, and M. Takayasu: "Electromechanical Investigation of 2 G HTS Twisted Stacked-Tape Cable Conductors," IEEE Trans. on Appl. Supercond., vol. 24 (3), pp.1-5, June, art. no. 6600405, 2014.

[17] D. Uglietti, et al.: "Development of HTS Conductors for Fusion Magnets," IEEE Trans. on Appl. Supercond., vol. 25 (3), pp.1-6, June, art. no. 4202106, 2015.

[18] D. C. van der Laan, et al.: "Correlation Between In-Plane Grain Orientation and the Reversible Strain Effect on Flux Pinning in RE-Ba2Cu3O7- $\delta$ Coated Conductors," IEEE Trans. on Appl. Supercond., vol. 22 (1), pp.1-7, Feb., art. no. 8400707, 2012.

[19] N. C. Allen, L. Chiesa and M. Takayasu: "Combined Tension-Torsion Effects on $2 \mathrm{G}$ REBCO Tapes for Twisted Stacked-Tape Cabling," IEEE Trans. on Appl. Supercond., vol. 25 (3), pp.1-5, June., art. no. 4800805, 2015.

[20] H.-S. Shin and M. Dedicatoria: "Review of progress in electromechanical properties of REBCO coated conductors for electric device applications," Prog. in Supercond. and Cryog. (PSAC), vol. 16 (4), pp. 7-16, 2014.

[21] Available on line at http://www.superox.ru/en/products/42-2G-HTS-tape/, accessed Sept. 4th, 2015.

[22] B. G. Falzon, and M. H. Aliabadi: "Buckling and postbuckling structures," World Scientific, 2008.

[23] L. Kollár and E. Dulácska: "Buckling of shells for engineers," John Wiley \& Sons, 1984. 